\title{
Fluid dynamic design of an axial rotor for hydrokinetic riverbed turbine - Improvement introduced by \\ a high lift foil profile
}

\author{
Oller, S. A. ${ }^{1 \dagger}$; Nallim, L. G. ${ }^{1}$; Oller, S. $^{2}$ \\ ${ }^{1}$ INIQUI - CONICET -Faculty of Engineering, National University of Salta, 440, Salta, Argentina \\ ${ }^{2}$ UPC-CIMNE - International Center for Method in Engineering, UPC Technical University of Catalonia \\ (Barcelona Tech), Barcelona 08034
}

†Corresponding Author Email: sergio.oller@conicet.gov.ar

\begin{abstract}
The main objective of this paper is to achieve a very high lift rotor to take the maximum advantage of the kinetic energy of a slow velocity water flow, which belongs to a lowland river type. Low speed flux and lack of depth are the main obstacles in hydrokinetic operation. The use of a high lift aerodynamic profile and the gain of the rotor number of blades serve to accomplish the task.

This work presents the fluid dynamic design for an axial hydrokinetic turbine rotor, studied in a three-dimensional (3-D) numerical simulation by means of Computational Fluid Dynamics (CFD). The use of CFD techniques avoids some physical model assays.

For the hydrokinetic turbine rotor design, first a one-dimensional (1-D) theoretical design was carried out, starting with the selection of a suitable airfoil profile to create the hydrofoil blade. Then, the 3-D rotor geometry was defined and studied
\end{abstract}


carefully by means of CFD, in order to check its hydrodynamic behaviour, i.e., lift and drag, streamline velocities and pressure fields. The CFD results were obtained using an open CFD code (Kratos).

Novelty: Despite hydrokinetic energy conversion is not a new technic; the application in a free water stream is becoming popular in the present. Most of the advances in this field involves oceanographic tides, relegating river flow usage to a very few studies. A very small part of these ambit concerns about a riverbed location of the hydrokinetic turbine, working in axial flow mode. Classical 3 bladed rotors in a drag operation enclose the advances in the field. This work aims to present the feasibility study of an 8 bladed hydrokinetic turbine rotor, improved by a high lift hydrodynamic profile.

Keywords: Hydrofoil, Hydrokinetic Generation, Water Current Turbine, Computational Fluid Dynamics (CFD).

\section{NOMENCLATURE}

C Airfoil chord (m)

c Relative flow velocity

$c_{y} \quad$ Lift coefficient

$c_{x} \quad$ Drag coefficient

$K L \quad$ Ratio between $\mathrm{X}$ and $\mathrm{L}_{\mathrm{avg}}$

$L_{\text {avg }}$ Chord average

$L_{x} \quad$ Airfoil's chord for each $\mathrm{x}(\mathrm{m})$

$P \quad$ Pressure $(\mathrm{Pa})$

Re Reynolds number 
SP Shape factor

$S P_{x} \quad$ Shape factor for each $x$

$S R_{x} \quad$ Non-dimensional parameter

$T \quad$ Torque $(\mathrm{Nm})$

$t \quad$ Time (s)

TSR Ratio between $c$ and $u$

$u \quad$ Blade linear rotational speed $(\mathrm{m} / \mathrm{s})$

$v \quad$ Absolute flow velocity $(\mathrm{m} / \mathrm{s})$

W Power $(\mathrm{w})$

$\omega \quad$ Angular speed $(\mathrm{rad} / \mathrm{s})$

$x \quad$ Length of each hydrofoil segment (m)

$X \quad$ Wingspan (m)

$Z \quad$ Number of blades of the rotor

$\alpha_{0} \quad$ Design attack angle $\left({ }^{\circ}\right)$

$\alpha \quad$ Real angle of attack $\left({ }^{\circ}\right)$

$\alpha_{\max }$ Maximum aerodynamic profile's $\alpha\left(^{\circ}\right)$

$\beta \quad$ Hydrofoil's camber angle $\left({ }^{\circ}\right)$

$\lambda \quad$ TSR

$\eta_{v} \quad$ Velocity tolerance convergence error

$\eta_{P} \quad$ Pressure tolerance convergence error

$\rho \quad$ Fluid density $\left(\mathrm{Kg} / \mathrm{m}^{3}\right)$

$\theta \quad$ Hydrofoil's sustentation angle $\left({ }^{\circ}\right)$ 


\section{Introduction}

Hydrokinetic generation [1] has become a very popular research topic.

Environmentally friendly, this is a type of clean energy capable of operating wherever natural water flow exists [2]. Despite these benefits, hydrokinetics in river operation is a much-unprofited energy due to the size of the turbine, which is limited by the river depth, and the low velocity of the flow [3].

River hydrokinetic energy conversion (HKEC) developers need site velocity and turbulence information over the energy extracting plane of their device for component design and estimation of power curves, annual energy production, and cost of energy. Measurements of mean velocity and Reynolds stress profiles, however, are not easily obtained for large rivers with depths exceeding one meter and currents greater than $1 \mathrm{~m} / \mathrm{s}$ (characteristics of the sites being targeted by the HKEC industry), given the challenges of deploying instruments under these hydrodynamic conditions. Rivers can have extreme variations in flow and stage, and measurements on the order of several decades are typically required to obtain meaningful statistics on the flow variability [4]. Neary et al [5] presented a study that reviews mean velocity and turbulence intensity profiles reported in the literature for open-channel flows to gain a better understanding of the range of current magnitudes and longitudinal turbulence intensities that these technologies may be exposed to. They compare 47 measured vertical profiles of mean current velocity and longitudinal turbulence intensity (normalized by the shear velocity) that have been reported for medium-large rivers, a large canal, and laboratory flumes with classical models developed for turbulent flat plate boundary layer flows. The comparison suggests that a power law and a semi-theoretical 
exponential decay model can be used to provide first-order approximations of the mean velocity and turbulence intensity profiles in rivers suitable for current energy conversion. In the same paper Neary et al [5] also shown the effect of river depth variation on the vertical location and size of the energy capture area for the deployment scenarios considered. In the present work, a $2 \mathrm{~m} / \mathrm{s}$ nominal water velocity [6] and around $10 \mathrm{~m}$ deep has been chosen to validate the proposed methodology.

Hydrokinetic technology can be affected by debris, sediment, surface ice, river dynamics (turbulence, current velocity, channel stability), and the interaction of turbine operations with fish and their habitat. The question of turbine operation impacts on the aquatic environment is one of the major issues that will determine stakeholder and permitting agency views toward this new technology [7].

Positioning the energy capture area closer to the bed would cause undesirable effects, including reducing the available power that can be harvested in the water column, increasing hydrodynamic load asymmetries and turbulence levels, and increasing the risk of strike or fouling by sediments or mobile bed forms [5]. The majority of the most advanced research in this area corresponds to a marine working condition [8], where rotors are available to reach 4 to 8 times the common lowlands river depth. In order to fill the gap, a hydrokinetic turbine for riverbed operation is being designed by the authors. Fluid dynamics studies of this turbine rotor are presented in this work. Starting out from a high efficiency tested hydrofoil [9] for geometry creation, the rotor hydrodynamic behaviour [10] at turbine working operation is studied in a river section. The study also takes into account the input conditions of a standard large-medium sized lowland river. The 
structural study of this rotor [11], engineered in composite materials, allows low inertia and functionality at low speed currents from fluvial beds.

\section{Hydrofoil and Rotor}

The basis of any machinery design is to obtain power. Obtaining this power $(W)$ is directly proportional to the machine's operating angular speed $(\omega)$ and torque (T) produced at that specific speed, as shown in Eq.(1),

$$
W=T \cdot \omega
$$

Torque and angular velocities are achieved by the hydrofoil's lift forces. If more lift is obtained by one blade, more torque and angular velocity will be obtained. This commitment is achieved by selecting the S1223 foil [12], which belongs to the high lift low Reynolds profiles class (see Fig. 1 from the University of Illinois Urbana-Champaign Airfoil Coordinates Database). Initially designed as an airfoil for air working conditions, the S1223 profile has also been tested by the authors as a hydrofoil under water conditions operation [9].

\subsection{Analytical Design}

\subsubsection{Previous hydrokinetic rotor studies}

The benefits of multi-blades rotors (more than three blades) relative to axial flows is that they have self-starting capabilities [13] [14], and offers a better power-dimensions ratio. These benefits, combined with the profile S1223 hydrofoil, have allowed us to conduct an investigation of various rotors with different numbers of blades. It began with an initial design of 29 blades with an outer ring to provide increased rigidity to the rotor (Figure 2a). For this case, the results showed a great disturbance in the fluid by the presence of the stiffener ring, 
which causes an alteration of velocity field, as shown in Figure 2b) as well as the forms taken by current lines at the ends of the hydrofoils.

After various trials combining different geometric elements of the rotor, we arrive at a configuration of 36 blades in a camber angle configuration of $\beta=10^{\circ}$, without stiffening ring (Figure 3a) which gives very good results in the velocity field (Figures 3b), stability in streamlines (Figure 4a) and very good balance of pressure between the upper and lower surface (Figure 4b). However, there are serious problems with the pressure distribution along the entire wingspan. In Figure 5 it is possible to observe how the lower surface has negative pressures near the axis, and the upper surface loses suction capacity in the periphery area. All greatly compromises the fluid dynamics stability of the rotor, relegating it into a non-optimal situation.

From experience and results obtained with different rotors, an eight-bladed rotor with S1223 hydrofoil profile is adopted. The proposed rotor keeps the proper balance between the lift and drag that the S1223 profile provides, and maintains an attached flow in the hydrofoil neighborhood (verified numerically). In consequence, this rotor has a better pressure distribution and presents hydrodynamic stability, preventing interference with the rest of the hydrofoils forming the rotor.

\subsubsection{Proposed hydrokinetic rotor design}

For turbine application, hydrofoil must be designed starting from the premise that it has to maintain fluid mechanics parameters (such as angle of attack, homogeneous pressure distribution, etc.) along the whole wingspan, despite the 
fact that rotary operating conditions produce different linear velocity of rotation (u) along the blade axis (which gets higher the nearer the point is from the wingtip).

Working with this condition involves the variation of the blade geometry parameters (like camber angle, airfoil chord, etc.) in relation with the wingspan axis. Figure 6 shows the notation used for angles and velocities on the blade profile, where $v$ is the absolute flow velocity, $u$ represents the blade's linear rotational speed and $c$ is the relative flow velocity.

The angle of attack $\alpha$ is an aero-hydrodynamic angle, defined between $c$ and the airfoil chord, and depends on the airfoil profile and its camber angle $\beta$. Instead, camber angle $\beta$ represents a mechanical angle, defined between the hydrofoil chord and its plane of rotation. By combining hydrodynamics and mechanical angles, a conceptual type of angle appears: the sustentation angle $(\theta)$, which is very useful to obtain the variating camber angle in a rotating blade.

Parameters involving the use of a S1223 profile working as a non-twisted, nonrotatory and unturbined designed hydrofoil are carried out by [9]. The suitable angle of attack occurs at the optimum angle of attack $\alpha_{0}=10^{\circ}$, which is considered as a starting parameter of the design sequence; it involves lift coefficient $c_{y}=2.2$ and drag coefficient $c_{x}=0.046$. Lift coefficient can raise until it reaches its maximum at $\alpha_{\max }=15^{\circ}$, but beyond that angle, detachment of the boundary layer will happen, dropping lift coefficient and increasing drag coefficient enormously [15]. 
The Tip Speed Ratio (TSR or $\lambda$ ) is a non-dimensional parameter that is defined by taking the relationship between the absolute axial flow velocity $c$ (river flow) and the blade speed $u$ (turbine rotor), and it is given by

$$
\lambda=(\omega \cdot R / v)
$$

According to Betz's law [16] (Eq.(10)), turbine mechanical power, specified for axial turbines (see Eq. (3) below) depends on the flux density ( $\rho$ ) and flow speed $(v)$; both values are fixed by the river flow, and so these parameters are fixed as initial conditions and will not be modified during the process of the rotor design. According to this, rotor nominal power is established in $30 \mathrm{KW}$, and is computed from

$$
W=\left(8 \cdot \rho \cdot A \cdot v^{3}\right) / 27
$$

The swept area $(A)$ is the unique variable in Eq. (3), and it depends on the radius (R) of the rotor.

As stated in Section 1 a medium/large-sized river with water velocity $v \cong 2 \mathrm{~m} / \mathrm{s}$ and around $10 \mathrm{~m}$ deep is used for the analysis. Based on these parameters, a standard hydrokinetic rotor turbine with a radius $R=2 m$ (Fig. 7), will be capable of operate in the majority of the many lowland riverbeds with a minimum of $10 \mathrm{~m}$ of deepness and a minimum of $2 \mathrm{~m} / \mathrm{s}$ of flow velocity. Angular speed of $\omega=$ $20 \mathrm{rev} / \mathrm{min}$ implies a $T S R=\lambda=1.8$, which satisfies the standard TSR values for multiple bladed rotors [17].

Despite the rotating condition, it is necessary to maintain the angle of attack along the wingspan; this scenario permits to keep the rotor's fluid dynamic stability. These commitments are accomplished by varying the geometry parameters of the hydrofoil (chord and camber $(L, \beta)$ ), along the wingspan (see Table1). To achieve 
this goal the Blade Element Theory is used; according to Froude [18, 31], the airfoil's total longitude $(X)$ is split in several sections, and each one is designed individually as $x$ (Fig. 8). Sustentation angle $\theta$ (Fig.6) is obtained by means of Eq. (4), as follows:

$$
\theta=\operatorname{acotg}((\omega \cdot x) / v)
$$

The chord of the airfoil is therefore computed for each segment $\left(L_{x}\right)$ by

$$
L_{x}=\left(S P_{x} \cdot x\right) /\left(c_{y} \cdot z\right)
$$

where $z$ is the actual number of airfoils in the rotor, $c_{y}$ is the lift coefficient corresponding to a defined profile section at a certain radius $x$, and the airfoil shape factor $S P_{x}$ can be computed by a curve approximation given by

$$
S P_{x}=2.2762 \cdot\left(S R_{x}^{-1.323}\right)
$$

In Eq. (6) the non-dimensional parameter $S R_{x}$ is given by

$$
S R_{x}=(T S R \cdot x) / X
$$

As a result of the chord modification during the process by Eq. (5), the initial attack angle $\left(\alpha_{0}\right)$ has to be recalculated too through Eq. (9), obtaining a new angle of attack $\alpha_{n}$ for each chord $\left(L_{x}\right)$ in each section $(x)$. For this recalculation, the $K L$ parameter, which represents a relationship between the wingspan and the average of the chord $\left(L_{a v g},\right)$ is necessary,

$$
K L=R / L_{\text {avg }}
$$

As the camber and the chord have been modified for each wingspan segment the angle of attack must be verified for each section through the following expression:

$$
\alpha_{n}=-\alpha_{0}+\left(c_{y} / 0.11\right) \cdot(1+(3 / K L))
$$


Solving from Eq. (2) to Eq. (9), the airfoil parameters are obtained as shown in Table 1.

Parameters in Table 1 allow the definitive design of the turbine blade as depicted in Fig. 9.

Blade parameters are strictly germane with the rotor composition through Eq.(10), which represents the ideal number of blades $(Z)$ presents in the rotor (according to flow and geometry parameters). The higher the number of blades present in the rotor, the higher the torque obtained will be. This condition also simplifies the starting of the turbine, ergo is a good design requirement, to have the more possible number of hydrofoils in the rotor.

$$
Z=\left(S P_{x} \cdot x\right) /\left(L_{x} \cdot c_{y}\right)
$$

\section{Numerical 3D simulation}

\subsection{Model geometry}

Bidirectional interaction between river and turbine is not studied in this work, so future study of the consequences in the river's behaviour at turbine working operation is proposed. As this work studies the turbine's behaviour in river motion, an ideal representation of the working operation is represented. The model is a confined fluid domain rendering the underwater operation without free surface. The 8-hydrofoil rotor is located inside a control volume that is made from a box of $8.5 \mathrm{~m} \times 12 \mathrm{~m} \times 8.5 \mathrm{~m}$, as shown in Fig. 10. These dimensions are chosen because a steady flow is needed at the boundaries of the box. Flow with $y$ direction will cross from the inlet surface to the outlet surface. Wall condition is given for the rest of domain surfaces. 


\subsubsection{Governing Equations and Numerical Approach}

Fluid mechanics governing equations for incompressible flows $(\rho=c t e)$ involves mass conservation condition (Eq. (11)), and Navier-Stokes equation (Eq. (12)):

$$
\begin{aligned}
& \nabla \cdot \vec{V}=0 \\
& \rho \frac{D \vec{V}}{D t}=\rho \vec{g}-\nabla P+\mu \nabla^{2} \vec{V}
\end{aligned}
$$

where $P$ is the pressure, $\vec{V}$ is the velocity field, $\rho$ is the fluid density, $\mu$ is the fluid viscosity, and $\vec{g}$ is the gravitational acceleration value.

Notice that this governing system is constituted by four equations and four unknowns, which are pressure and the three vector components of the field velocity; so numerical techniques are necessary for this treatment and, in this particular case, a Finite Element Variational Multiscale Simulation method (FEVMS) [19][20] is applied as the resolution method.

Close to FEVMS method that uses three numerical scales of turbulence structures (large resolved, small resolved and smaller unresolved) is the Large Eddy Simulation (LES) [21] [22] method. This method uses two scales of turbulence structures (resolved and unresolved). The solver (with general equations of fluid given by Eq. (12)) solves the larger turbulent structures, while the energy dissipation of the minor scales takes place through any turbulent model. The threshold that defines what the solver solves and what the turbulent model employs is decided by the user through a tolerance. Also there are classical turbulence models (e.g. Ref. [23]) that use to solve the smaller unresolved scales. In fact, FEVMS uses the finite element space grid of the mesh to solve the resolved scales. So with a very small space grid and a fine $\Delta t$ stabilization, the 
method is capable of controlling the influence of the smaller scales on the bigger ones, closer to a Direct Numerical Simulation (DNS) (see for instance [24]), but with the same computational cost as a LES model.

\subsubsection{Meshing criteria}

The computational domain has a length of $12 \mathrm{~m}$, width of $8.5 \mathrm{~m}$ and depth of $8.5 \mathrm{~m}$ as shown in Figure 10. The control volume mesh for the simulation of fluid, as is presented in this paper, was generated from two basic geometries. One such geometry is defined by the limits of the control volume (outer limits in Figure 11), and inner limits defined by the finite element mesh used for the structural analysis of the rotor [11]. An 80,000 linear tetrahedral elements [25] [26] mesh is generated. This type of tetrahedron mesh is chosen since it is capable of meshing complex geometries. The mesh is 10 times denser in the vicinity of the rotor, which is where interested in studying the velocity fields, pressure, streamlines, etc.

Figure 11 shows in more detail the generated finite element mesh for the control volume and for the complete rotor. From a general point of view it gives the idea of its complexity, because it displays details that are also represented in the finite element discretization. In these details, it is possible to observe the relationship of scales have been taken into account to perform this fluid dynamic analysis.

\subsubsection{Boundary conditions and solver usage}

The fluid condition involves water density, water viscosity, and a forced velocity field in the inlet surface with a modulus of $2 \mathrm{~m} / \mathrm{sin} y$ direction. Flow crosses the 
control volume to the outlet surface, where a fix pressure condition is given. Except for inlet and outlet surfaces, all other model surfaces have a No-Slip velocity condition assigned.

The numerical conditions used in this incompressible problem type involves a simulation runtime of $t=10 \mathrm{~s}$, which is needed to achieve steady flow; a solver using a BiConjugate gradient stabilized [27] for the velocity field calculations; and a Skyline LU factorization [28] direct solver type, for pressure field resolution.

Solver runs under $16 \mathrm{MB}$ of RAM i5 -3.30GHz Intel CPU, and it takes 4 days for the process finish to be achieved in a convergence criterion of 3 iterations per time step, involving $\Delta t=1 \mathrm{eq} \cdot 10^{-2} \mathrm{~s}$

\section{Results}

GID [29] post processor is launched with KRATOS [30] problem type and postprocessing results are presented next.

Despite the high Reynolds number reached by the blades of the rotor, because of the fluid density, streamlines results display a non-detached flow in the blades of the rotor (Fig. 12c, Fig. 12d), and also a steady flow all over the studied control volume (Fig. 12a). Figure 12b manifests the wingtip vortex phenomenon, whose treatment is previewed as a future line.

Achieving nearly $3.2 \mathrm{~m} / \mathrm{s}$ of flow velocity in the upper surface of the hydrofoil (Fig. 13), and1.4 m/s of flow velocity in the lower surface (Fig. 13), the phenomena produces relative pressure values of $P_{\text {Outtersurface }}=-1600 \mathrm{~Pa}$ (Fig. 14 ) in the upper surface, and $P_{\text {Innersurface }}=3700 \mathrm{~Pa}$ in the lower surface (Fig. 
15); generating a lift capacity corresponding to $\Delta P_{\text {blade }}=5400 \mathrm{~Pa}$ for each blade. Figure 14 and Figure 15 also display a homogeneous distribution of the pressure all over the wingspan axis; this data is relevant in a turbine hydrofoil, whose chord measurement and camber angle are not constant along the blade. It is important to point out that numerical simulation shows no boundary layer detachment, and also displays a smooth and continuous pressure distribution [31] all over the upper and lower surfaces of the turbine blades.

\section{Conclusions}

The particular designed eight-bladed hydrokinetic rotor presented in this work reaches the initial proposed goal; working with $8 \cdot 10^{6}$ Reynolds number, it is capable of obtaining $30 \mathrm{~kW}$ of mechanical power, demonstrating its operating ability imbibed in a low velocity axial flow (lowland riverbeds flows). Besides achieving high efficiency in a low speed flux usage, it also keeps a stable behaviour of the streamlines crossing the control volume. The particular blade, designed on the basis of a high lift profile, originally oriented for aerodynamics tasks, reaches an average of $1500 \mathrm{~N}$ of lift force, in a working configuration of $\alpha=14^{\mathrm{o}}$ of angle of attack. Through the numerical simulation a smooth and continuous pressure distribution all over the outer and inner surfaces of the turbine blades are shown, making hydrofoil and rotor design suitable for hydrokinetic riverbed turbine usage.

Finally, it is important to remark that we consider the CDF procedure enough for fluid dynamic designing and for analysing viability of the hydrokinetic turbine proposed. Moreover in this analysis a "weak-coupling" fluid-structure (FSI) has 
been considered. This interaction allows reaching the previous structural design proposed by the authors. Also this procedure gives feasibility to our hydrokinetic turbine rotor improved by the high lift hydrodynamic profile.

\section{Acknowledgements}

This work has been supported by Barcelona Tech (Technical University of Catalonia UPC), Spain; International Center for Numerical Method in Engineering (CIMNE), Spain; CIMNE-Classrooms of National University of Salta, Argentina; Consejo Nacional de Investigaciones Científicas y Técnicas (CONICET), Argentina, and Consejo de Investigación de la Universidad Nacional de Salta (CIUNSa), Argentina. All this support is gratefully acknowledged.

\section{References}

[1] Khan, M.J., Iqbal, M.T., Quaicoe, J.E. (2008). River current energy conversion systems: Progress, prospects and challenges. Renewable and Sustainable Energy Reviews 12, 2177-2193.

[2] Kaya, A.M., Kandemir, I., Akşit, M.F., Yiğit, K.S. (2015). Investigation of Optimum Working Conditions of a Micro Cross Flow Turbine. Environmental Progress \& Sustainable Energy (Vol.00, No.00) DOI 10.1002/ep.12112

[3] Khan, M.J., Bhuyan, G., Iqbal, M.T., Quaicoe, J.E. (2009). Hydrokinetic energy conversion systems and assessment of horizontal and vertical axis turbines for river and tidal applications: A technology status review, Applied Energy 86, 1823-1835. 
[4] Neary, V.S. and D.C. Sale. (2010). Flow characteristics of river resources for hydrokinetic energy conversion, in Hydrovision 2010. 2010: Charlotte, NC.

[5] Neary, V.S., Gunawan, B. Sale, D.C. (2013). Turbulent inflow characteristics for hydrokinetic energy conversion in rivers. Renewable and Sustainable Energy Reviwes 26, 437-445.

[6] Muñoz, A.H., Chiang, L.E., De la Jara, E.A. (2014). A design tool and fabrication guidelines for small low cost horizontal axis hydrokinetic turbines. Energy for Sustainable Development 22, 21-33.

[7] Johnson, J.B. , Pride, D.J. (2010). River, Tidal, and Ocean Current Hydrokinetic Energy Technologies: Status and Future Opportunities in Alaska. Prepared for the Alaska Energy Authority By the Alaska Center for Energy and Power, November 1, 2010.

[8] Güney, M.S., Kaygusuz, K. (2010). Hydrokinetic energy conversion systems: A technology status review. Renewable and Sustainable Energy Reviews 14, 2996-3004.

[9] Oller, S.A., Nallim, L.G., Oller, S. (July 2016). The usability of the Selig S1223 profile airfoil as a high lift hydrofoil for hydrokinetic application. Journal of Applied Fluid Mechanics 9(3). ISSN 1735-3572. Acepted paper in press.

[10] Hossein, B.A.,Bibeau, E.L., Chatoorgoon, V., Kumar, A. (2013). Power measurement of hydrokinetic turbines with free-surface and blockage effect. Ocean Engineering 69, 9-17. 
[11] Oller, S.A., Nallim, L.G., Oller, S. (2013). An Integrated procedure for the structural design of a composite rotor-hydrofoil of a water current turbine (WTC). Applied Composite Materials 20(6), 1273-1288.

[12] Selig, M.S., Guglielmo, J.J. (1997). High-Lift Low Reynolds Number Airfoil Design. Journal of aircraft 34(1), 72-79.

[13] Kolekar N., Hu Z., Banerjee A., Du X. (2013). Hydrodynamic Design and Optimization of Hydro-kinetic Turbines using a Robust Design Method. Proceedings of the 1st Marine Energy Technology Symposium METS13. April 10-11, 2013, Washington, D.C.

[14] Ismail A.A.R., Batalha. A comparative study on river hydrokinetic turbines blade profiles. International Journal of Engineering Research and Applications. Vol. 5, Issue 5, (part - 1) May 2015, pp. 01-10.

[15] Goundar, J.N., Rafiuddin Ahmed, M., Lee, Y.-H. (2012). Numerical and experimental studies on hydrofoils for marine current turbines. Renewable Energy 42, 173-179.

[16] Betz, A. (1966). Introduction to the Theory of Flow Machines, Oxford, New York: Pergamon Press.

[17] Amelio, M., Barbarelli, S., Florio, G., Scornaienchi, N.M., Cutrupi, A., Minniti, G., Sánchez Blanco, M. (2012). Innovative tidal turbine with central deflector for the exploitation of river and sea currents in on-shore installations. Applied Energy 97, 944-955.

[18] Froude, W. (1878). On the elementary relation between pitch, slip an propulsive efficiency. Transactions of the Royal Institute of Naval Architects. 
[19] Hughes, T. (1995). Multiscale phenomena, Green's functions, the Dirichletto-Neumann formulation, subgrid-scale models, bubbles and the origin of stabilized methods. Computer Methods in Applied Mechanics and Engineering 127, 387-401.

[20] Guermond, J. (1999). Stabilization of Galerkin approximations of transport equations by subgridmodeling. Mathematical Modelling and Numerical Analysis 33, 1293-1316.

[21] Hughes, T., Mazzei, L., Jansen, J. (2000). Large Eddy simulation and the variation multiscale method, Computing and Visualization in Science 3, 4759.

[22] Sagaut, P. (2006). Large Eddy Simulation for Incompressible Flows, 3rd ed. Berlin, Heidelberg, New York: Springer-Verlag.

[23] Smagorinsky, J. (1963). General circulation experiments with the primitive equations. Monthly Weather Review 91, 99-152.

[24] Moin, P., Mahesh, K. (1998). Direct numerical simulation- A tool in turbulence research. Annual Review of Fluid Mechanics 30, 539-578.

[25] Zienkiewicz, O.C., Taylor, R.L. (2000). The finite element method, volume III: Fluid dynamics. Butter Worth Hinemann. 5th edition.

[26] Lewis, R., Nithiarasu, P., Setharamu, K. (2004). Fundamentals of the finite element method for heat and finite element method for heat and fluid flow, John Wiley and sons.

[27] Van der Vorst, H.A. (1992). Bi-CGSTAB: A Fast and Smoothly Converging Variant of Bi-CG for the Solution of Nonsymmetric Linear Systems. SIAM Journal on Scientific Computing 13, 631-644. 
[28] Felippa, C.A. (1975). Solution of linear equations with skyline-stored symmetric matrix. Computers \& Structures 5(1), 13-29.

[29] GID (1996). The personal pre and post processor, CIMNE. Available: http://www.gidhome.com/.

[30] Kratos (2000). Multi-Physics, CIMNE. [Online]. Available: http://www.cimne.com/kratos/.

[31] Wang, J.-F., Piechna J, Müller N(2012). A novel design of composite water turbine using CFD. Journal of Hydrodynamics 1(24),11-16.

[32] Carlton J. S. (2012). Marine propellers and propulsion. BH Elsevier Third Edition 